\title{
Hansel, Toni
}

\section{Ganztagsschule - Angebot für eine Optimierung von \\ Unterrichtsversorgung und Unterrichtsqualität}

Hansel, Toni [Hrsg.]: Ganztagsschule. Halbe Sache - großer Wurf? Schulpädagogische Betrachtung eines bildungspolitischen Investitionsprogramms. Herbolzheim : Centaurus 2005, S. 20-32. (Schulpädagogik; 7)

Quellenangabe/ Reference:

Hansel, Toni: Ganztagsschule - Angebot für eine Optimierung von Unterrichtsversorgung und Unterrichtsqualität - In: Hansel, Toni [Hrsg.]: Ganztagsschule. Halbe Sache - großer Wurf? Schulpädagogische Betrachtung eines bildungspolitischen Investitionsprogramms. Herbolzheim : Centaurus 2005, S. 20-32 - URN: urn:nbn:de:0111-opus-34253 - DOI: 10.25656/01:3425

https://nbn-resolving.org/urn:nbn:de:0111-opus-34253

https://doi.org/10.25656/01:3425

in Kooperation mit / in cooperation with:

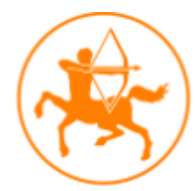

\section{CENTAURUS}

\section{Verlag \& Media KG}

\section{Nutzungsbedingungen}

Gewährt wird ein nicht exklusives, nicht übertragbares, persönliches und beschränktes Recht auf Nutzung dieses Dokuments. Dieses Dokument ist ausschließlich für den persönlichen, nicht-kommerziellen Gebrauch bestimmt. Die Nutzung stellt keine Übertragung des Eigentumsrechts an diesem Dokument dar und gilt vorbehaltlich der folgenden Einschränkungen: Auf sämtlichen Kopien dieses Dokuments müssen alle Urheberrechtshinweise und sonstigen Hinweise auf gesetzlichen Schutz beibehalten werden. Sie dürfen dieses Dokument nicht in irgendeiner Weise abändern, noch dürfen Sie dieses Dokument für öffentliche oder kommerzielle Zwecke vervielfältigen, öffentlich ausstellen, aufführen, vertreiben oder anderweitig nutzen.

Mit der Verwendung dieses Dokuments erkennen Sie die Nutzungsbedingungen an.

\section{Kontakt / Contact:}

\section{peDOCs}

DIPF | Leibniz-Institut für Bildungsforschung und Bildungsinformation Informationszentrum (IZ) Bildung

E-Mail: pedocs@dipf.de

Internet: www.pedocs.de

\section{Terms of use}

We grant a non-exclusive, non-transferable, individual and limited right to using this document.

This document is solely intended for your personal, non-commercial use. Use of this document does not include any transfer of property rights and it is conditional to the following limitations: All of the copies of this documents must retain all copyright information and other information regarding legal protection. You are not allowed to alter this document in any way, to copy it for public or commercial purposes, to exhibit the document in public, to perform, distribute or otherwise use the document in public.

By using this particular document, you accept the above-stated conditions of use. 


\section{GANZTAgSSCHULE. Halbe Sache - grober Wurf?}

Schulpädagogische Betrachtung eines bildungspolitischen Investitionsprogramms

Toni Hansel (Hg.)

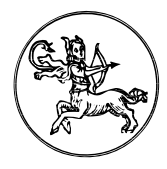

Centaurus Verlag

Herbolzheim 2005 
Der Herausgeber, Prof. Dr. Toni Hansel, ist Professor für Schulpädagogik an der Universität Rostock und Direktor des Instituts für Schulpädagogik.

Die Deutsche Bibliothek - CIP-Einheitsaufnahme

Bibliographische Information der Deutschen Bibliothek Die Deutsche Bibliothek verzeichnet diese Publikation in der Deutschen Nationalbibliographie; detaillierte bibliographische Daten sind im Internet über http://dnb.ddb.de abrufbar.

ISBN 3-8255-0614-2

\section{ISSN 1616-7414}

Alle Rechte, insbesondere das Recht der Vervielfältigung und Verbreitung sowie der Übersetzung, vorbehalten. Kein Teil des Werkes darf in irgendeiner Form (durch Fotokopie, Mikrofilm oder ein anderes Verfahren) ohne schriftliche Genehmigung des Verlages reproduziert oder unter Verwendung elektronischer Systeme verarbeitet, vervielfältigt oder verbreitet werden.

() CENTAURUS Verlags-GmbH \& Co. KG, Herbolzheim 2005

Umschlaggestaltung: Antje Walter, Hinterzarten

Satz: Vorlage des Herausgebers

Druck: primotec-printware, Herbolzheim 


\section{Disposition}

Grußwort des Dekans der Philosophischen Fakultät

Prof. Dr. Wolfgang Sucharowski

Schulpädagogische Betrachtung eines

bildungspolitischen Investitionsprogramms

$2.1 \quad$ Literatur

Eröffnung des Symposiums durch den Studiendekan der Philosophischen Fakultät

Prof. Dr. Wolfgang Nieke

Ganztagsschule als Bestandteil von Ganztagsbildung

\section{Perspektiven einer revitalisierten Reformidee}

Toni Hansel

Ganztagsschule - Angebot für eine Optimierung von Unterrichtsversorgung und Unterrichtsqualität

Ganztagsschule - nicht Erweiterung, sondern Vertiefung des

Schulzweckes

Voraussetzungen für die Einrichtung von Ganztagsschulen 
5.1 Reformpädagogik ohne Bedeutung für eine moderne Ganztagserziehung?

5.2 Beiträge der deutschen Reformpädagogik zu Konzeption und Realisierung moderner Formen der Ganztagsschule 39

$\begin{array}{lll}\text { 5.2.1 Die Bedeutung der deutschen Landerziehungsheime } & 39\end{array}$

5.2.2 Die Bedeutung der Wald- und Freiluftschulbewegung 43

5.2.3 Die Bedeutung der Arbeitsschulbewegung 48

5.2.4 Die Bedeutung der Schulpädagogik Herman Nohls 50

5.3 Reformpädagogisch gestaltete Ganztagsschule als Schule der Zukunft?

\section{Ganztagsschule und gesellschaftliche Vernetzung}

\section{Thomas Coelen}

Ganztagsbildung: Qualifikation und Partizipation von Kindern und Jugendlichen auf kommunaler Basis

6.1 Zur Idee hinter dem Begriff, Ganztagsbildung 57

6.2 Unterscheidung von Ganztagsschule, Ganztagsbetreuung und Ganztagsbildung

6.3 Anknüpfungen an gesellschaftstheoretisch gerahmte Bildungsbegriffe

Die Bedeutung der Ganztagsschule für den Wirtschaftsstandort Deutschland 


\title{
Ganztagsschule als bildungspolitische Option
}

\author{
Michael Becker/Ute Debold
}

Entwicklungsschub für Ganztagsschulen in

Mecklenburg-Vorpommern als landespolitische Aufgabe nach PISA

8.1

$\begin{array}{ll}\text { Einleitung } & 102\end{array}$

8.2

Die Ganztagsschule in gebundener Form

104

8.3

Das Evaluierungsprogramm Mecklenburg-Vorpommern

Probleme, Notwendigkeiten und Möglichkeiten dieser

Schulform

108

Ziele und Kriterien der pädagogischen Konzepte

Grundpositionen der Unterrichtsgestaltung an

Ganztagsschulen

Resümee

\section{Jörg-Dieter Gauger}

Ganztagsschule zwischen bildungspolitischer Opportunität und schulpädagogischer Konzeptlosigkeit

Zur Spannung zwischen Schulpädagogik und Bildungspolitik 


\title{
Neue Wege aus dem schulpraktischen Reformstau?
}

\author{
Angela Schulz
}

Ganztagsschulen und die richtige Antwort auf PISA: Ein Erfahrungsbericht

\section{Bernd Ostermeyer}

Ganztagsschule und Ganztagsbetreuung auf dem Prüfstand schulpraktischen Alltagshandelns

11.1 Jugend und Schule in veränderter Gesellschaft 152

11.2 Was kann und was soll Schule leisten? 154

11.3 Ganztagsschulen und Ganztagsbetreuung realistisch betrachtet 157

11.4 Zum Umgang mit dem 'Investitionsprogramm Zukunft, Bildung und Betreuung im schulischen Alltag'

\section{Eckhardt Preuß}

Leistungserziehung und Leistungsbeurteilung in der Grundschule/Schule im Blick auf die Ganztagsschule

12.1 Vorbemerkung zur Problematik und Inhalt des Themas

Die Welt der Kinder heute als Herausforderung für die Neugestaltung von Schule (Halbtags-Ganztagsschule) und Unterricht im Blick auf den Baustein „Leistungserziehung und Leistungsbeurteilung“

12.3 Pädagogisches Sehen und „pädagogisches Verstehen“ (W. Klafki ) - kritische Anmerkungen zu den alten und neuen Grundschulrichtlinien von NRW 1985 und 2003

12.3.1 Zentrale Aussagen der Richtlinien von 1985 anhand der „doppelten Reckstange“

12.3.2 Zentrale Aussagen der Richtlinien 2003 anhand einer „normierten“ Reckstange“

12.4 Fünf grundlegende Ziele der Leistungserziehung in Korrespondenz zu den fünf Tätigkeiten des Kindes 173 
12.6 Pädagogische Leistungsbeurteilung im Sinne und in der Ausgestaltung von Lernentwicklungsberichten 177

12.7 Literatur

\title{
Kulturelle Netzwerke und schulische Verantwortung
}

\author{
Eckart Pankoke
}

„Offene Ganztagsschule“ und „Kulturen der
Verantwortung“. Netzwerke und Lernprozesse
schulischer Selbststeuerung

Schul-Geschichte(n)

Neue Perspektiven und Horizonte

13.7 Selbständigkeit und Selbststeuerung der Organisations- und Relationsentwicklung

\section{Cristina Allemann-Ghionda}

Ganztagsschule internationalen Vergleich - von der Opposition zur Arbeitsteilung zwischen Staat und Familie? 
$\begin{array}{lll}\text { 14.3.3 } & \text { Erwerbstätige Mütter - akzeptiert } 209\end{array}$

14.3.4 Vorschulerziehung und Chancengleichheit 209

14.3.5 Täglicher Ablauf und staatliche Aufsicht 210

14.3.6 Kommentar 211

Pädagogische Argumente - kulturell gebunden oder
transnational?

14.4.1 Politische und soziale Argumente, psychologische und

14.4.2 Die Ganztagsschule in Italien 214

14.4.3 Ganztagsschule in der Schweiz 216

14.4.4 Ganztagsschule in Russland 217

$\begin{array}{lll}14.5 & \text { Schluss } & 219\end{array}$

14.6 Literatur 222

15 Toni Hansel

Was leistet Ganztagsschule? $\quad 224$

$15.1 \quad$ Vorbemerkung 224

15.2 Modellalternativen ganztagsschulischer Förderung 226

15.3 Welche Erwartungen verbinden sich mit der Einrichtung von Ganztagsschulen? 229

$15.4 \quad$ Wachsender Konsens 232

15.5 Was leistet die Ganztagsschule, was die Halbtagsschule nicht leisten kann? 235

15.6 Fazit 248

$\begin{array}{lll}15.7 & \text { Literatur } & 248\end{array}$

16 Autorenspiegel 250

$17 \quad$ Namensregister 


\section{Toni Hansel}

\section{Ganztagsschule - Angebot für eine Optimierung von Unterrichtsversorgung und Unterrichtsqualität}

\subsection{Vorbemerkung}

Das Klima für Veränderungen im Bildungswesen ist im Augenblick so günstig wie schon sehr lange nicht mehr. Dabei wird der Reform-Elan - um den Überschwang gleich im Keime zu dämpfen - keineswegs überall aus intrinsischer ReformMotivation getragen, sondern er ist, der Not gehorchend, ein Reflex auf das erschreckend schwache Abschneiden der deutschen Schülerpopulation in der internationalen Schulleistungsstudie PISA, die seit dem Jahr 2000 Unruhe in eine behäbig gewordene Bildungspolitik hineingetragen hat. Zwar gibt es kein plausibles, auch empirisch belastbares Argument für die These, dass einer der vielen Punkte des Bildungswesens, für die jetzt vehement Reformen eingefordert werden, wie z.B. das unterstellte Versagen des frühpädagogischen Bereichs, die Minderausstattung mit Ganztagsschulen, die Lehrerbildung usw. usw. kausal für das PISA-Desaster der 15jährigen deutschen Schüler verantwortlich zu machen ist. Hier sind gesamtsystemische Überlegungen sicher angebracht, aber die Initialzündung, die die Studie auslöste, entfachte eine Dynamik, die gelegentlich in das andere Extrem hinüberwogte: Aktionismus statt besonnene Aktion war ein häufig beobachtbares Phänomen in der Folge der PISA-Debatte. 
Zwei Dinge fallen in diesem Zusammenhang auf: Die Tendenz zeichnet sich ab, lediglich mehr von dem zu veranstalten, was uns in PISA als Defizit angerechnet wurde: mehr vom gleichen optimierungsbedürftigen Unterricht! Und es gehen die Meinungen darüber, was unter Unterricht zu verstehen ist, inzwischen so weit auseinander, dass sie kaum noch auf die gleiche Sache zu beziehen sind (GIESECKE 2004).

Die Ganztagsschule, mit der wir uns in diesem Band beschäftigen, ist keine Erfindung unserer Tage, sondern eine Einrichtung öffentlicher Bildung und Erziehung mit einer sehr langen, wenngleich auch nicht flächendeckenden Tradition in Deutschland, aber stärker noch im Ausland; sie kommt sowohl in privater als auch in öffentlicher Trägerschaft vor. Ihre derzeitige Aktualität ist aus den sich wandelnden Verhältnissen industrialisierter Gesellschaften unter Einbezug der gravierenden demographischen Veränderungen herzuleiten, die das bisherige Gefüge aus innerfamiliarer Versorgung und außerfamiliarer Bildung dynamisierten - ich kehre gleich noch einmal zu diesem Aspekt zurück. Die politischen Parteien der Bundesrepublik Deutschland haben mittlerweile alle die mehrfach dimensionierte politische Bedeutung und Brisanz dieser Bildungseinrichtung erkannt, kommen aber zu durchaus kontrastreichen Schlussfolgerungen.

Ich möchte zunächst eine Eingrenzung vornehmen und darauf hinweisen, womit ich mich in dieser Einführung nicht zu beschäftigen beabsichtige. Höchst interessant für den historischen Pädagogen ist ein Vergleich der Entwicklung in Deutschland und den angelsächsischen Ländern, die keineswegs einheitlich verlief; auch die reformpädagogischen Entwürfe - ich nenne hier nur einige Namen, z.B. Georg Kerschensteiner, Paul Oestreich, Wilhelm Ganzenmüller, Peter Petersen, Adolf REICHWEIN etc. etc. - sollen hier nicht abgearbeitet werde, weil es die bloße Wiederholung vorangegangener Diskussionen wäre. Harald LUDWIG (1993) hat darauf aufmerksam gemacht, dass die Diskussion um Ganztagsschulen in Deutschland auch immer eine Diskussion um reformpädagogische Ideen gewesen ist. Gerade des- 
halb ist es jedoch wichtig, am Beginn einer neuen Reforminitiative mit der zu Gebote stehenden Distanziertheit zu bilanzieren - eine solche Bilanz zieht er auch in seinem Beitrag zu diesem Band (vgl. Abschn. 5) und verknüpft sie mit den Schwerpunkten der aktuellen Ganztagsschuldebatte.

Nahe am Thema liegt da schon ein Vergleich der Positionen, die von politisch bzw. gesellschaftlich relevanten Gruppen im Zusammenhang mit der Ganztagsschule vertreten werden. Es liegt auf der Hand, dass der hier gewählte Begriff gesellschaftlich relevante Gruppe sehr schillernd und eigentlich nicht sonderlich präzise ist. Die Gruppe der fünf Millionen Arbeitslosen ist in volkswirtschaftlichem Kontext eine äußerst relevante gesellschaftliche Gruppe, in unserem thematischen Zusammenhang sind sie ohne Belang. Wir haben uns deshalb bemüht, politische, sozialwissenschaftliche, wirtschaftliche, kirchliche, nicht zuletzt auch schulpraktische Positionen (vgl. Schulz, Abschn 10; Ostermeyer, Absch. 11; Preub, Abschn. 12) hier zu erfassen - das Ensemble der Beiträge gibt dies alles wieder. Die aktuelle bildungspolitische Debatte, der Gemeinsames und Trennendes zwischen den politischen Lagern zu entnehmen ist - sind das Terrain, von dem aus Jörg-Dieter GAUGER sich dem Leitthema annähert. BECKER/DEBOLD beschreiben die bildungspolitische Umsetzung des vom Bund angestoßenen Bundesprogramms „Zukunft Bildung und Betreuung“" (IZBB) in Höhe von vier Milliarden Euro, davon 93 Millionen für Mecklenburg-Vorpommern, aus der Sicht des für dieses Vorhaben zuständigen Landesministeriums (Abschn. 8).

Schule ist keine Veranstaltung außerhalb der Gesellschaft, sondern nicht ablösbarer Teil von ihr, ja sie ist auf vielfältige Weise vernetzt mit ihren Teilsystemen. Die wirtschaftlichen Zusammenhänge erläutert Stefan KüPPER von der BdA (Abschn. 7). In der Tat gewinnt, wer die Debatte zur Ganztagsschule verfolgt, mitunter den Eindruck, dass es auch wesentlich um Fragen des Marktes, nämlich um Fragen des Arbeitsmarktes dabei geht: Eltern sind auch Arbeitnehmer, und wenn die „lieben Kleinen“ den Tag über in der Schule sind, dann stehen sie - die Eltern - dem Markt zur 
Verfügung. Die These ist nicht von der Hand zu weisen, dass Investitionen in Bildung sehr bedeutsame Zukunftsinvestitionen sind, von denen unser aller wirtschaftliches Wohl zunehmend abhängt. Um dies deutlich zu machen, vernetzt KÜPPER diesen Schultypus mit den Bedingungen, aber auch den Erwartungen des Wirtschaftsstandorts Deutschland und entwickelt in diesem Zusammenhang auch die Qualitätskriterien, die die deutsche Wirtschaft - im konkreten Fall in Übereinstimmung mit dem DGB - von diesem Schultypus erwartet; auch COELEN (vgl. Abschn. 6) unternimmt einen Versuch, die diffuse Debatte zu bündeln und dennoch nicht das Anliegen aus den Augen zu verlieren, nämlich Ganztagsbildung nicht in der Engführung von Lernleistungen und Vereinbarkeit von Familie und Beruf schulpädagogisch oder familienpolitisch zu regionalisieren, sondern durch die Vernetzung von Bildung und Jugendhilfe unter Wahrung ihrer Eigenheiten ein integriertes Ganzes hervortreten zu lassen.

Hier ist zunächst die Thematisierung schulpädagogischer Fragestellungen im Zusammenhang mit der Errichtung von Ganztagsschulen vorzunehmen. Bei dieser Aufgabe sekundiert mir Bernd OsTERMEYER (Abschn. 11), der als Leiter eines Ganztagsgymnasiums den Alltag dieses Schultyps von der Praxisseite her gestaltet und damit in all seinen Facetten kennt.

\subsection{Ganztagsschule - nicht Erweiterung, sondern Vertiefung des Schulzweckes}

Wer einen Blick in die Regelungswerke wirft, die die Bundesländer mit dem Ziel der inneren und äußeren Ausgestaltung und der rechtlichen Absicherung der Schule und des in ihr vollzogenen Handelns erlassen haben - also Richtlinien, Erlasse, Gesetze, Verordnungen etc. - der wird relativ rasch auf das die Bundesländer einende stoßen: den Schulzweck! Die Schule ist eine öffentlich-rechtliche Einrichtung, deren primärer Zweck die Bildung und Erziehung der nachwachsenden Generation einer 
Gesellschaft ist. Über die Wege, wie man diesen Schulzweck erfüllen kann, gibt es bekanntlich durchaus abweichende föderale Auffassungen, aber es gibt wohl einen Konsens darüber, dass Bildung und Erziehung nicht veräußerbare Zielvorgaben des Lehrerhandelns in der Schule sind. Auch die Ausweitung des Schulbetriebs auf den ganzen Tag verändert diesen Schulzweck nicht und vor diesem Hintergrund ist jede Veränderung des Schulbetriebs daraufhin zu befragen, wieweit sie diesem Schulzweck förderlich ist.

Die Veränderung des Schulbetriebes ist in diesem Zusammenhang aber nicht das einzige Veränderungspotential. Beispielhaft und gewiss nicht vollständig seien hier genannt:

\section{Demographische Veränderungen}

- Wandel der Familienkonstellation

- steigende Zahl der nichtehelichen Lebensgemeinschaften

- steigende Zahl der Ehescheidungen

- weiter ansteigende Tendenz zur Kleinfamilie

\section{Veränderungen des Wobnumfeldes}

- Verlust/Veränderung von Erfahrungsräumen in Familie und Wohnumwelt

- autogerechte Umgestaltung der Wohnumwelten

- Veränderung von Siedlungsformen

Nicht alle diese Veränderungsvariablen sind in einen unmittelbaren Zusammenhang mit der Schule zu bringen, aber sie haben einen mehr oder minder direkten Einfluss auf die Voraussetzungen, unter denen Schulen, also auch Ganztagsschulen eingerichtet werden. Insbesondere die demographischen Veränderungen haben den Schulzweck deutlich vertieft. Bildung und Eræziehung waren zuvor schon unumstrittene Zweckbindungen schulischen Wirkens, sie sind es heute auf eine neue Weise: Bil- 
dung ist angesichts des globalen ökonomischen Wandels zu einem Wettbewerbsfaktor von herausragender Zukunftsbedeutung geworden; Erziehung leistet die Schule in hohem Maße, aber dennoch unzureichend dort, wo elterliche Erziehungsleistungen aus welchen Gründen auch immer weggefallen sind bzw. wo die Tendenz zur Ein-Kind-Familie den Kindern grundlegende Familienerfahrungen vorenthält. Solcher Wandel wird neben anderen zentralen Aspekten in den Beiträgen von Eckart PANKOKE (Abschn. 13) und Cristina AlLEMANn-GHiOnda (Abschn. 14) vertieft.

\subsection{Voraussetzungen für die Einrichtung von Ganztagsschulen}

Die Einrichtung von Ganztagsschulen erfordert eine Reihe von Voraussetzungen, ohne deren Vorliegen die Ganztagsschule nur eine in die Länge gezogene Halbtagsschule ist. Damit wird auch deutlich, dass die Hürden für eine schulpädagogisch konturierte Ganztagsschule recht hoch liegen (BECKER/DEBOLD, Abschn. 8).

- Ganztagsschule bedeutet zugleich auch Ganztagsversorgung.

- Das Schulkonzept kann nicht additiv sein - alles wie bisher, nur etwas länger - sondern muss aufeinander abgestimmt sein. Vormittägliche und nachmittägliche Aktivitäten stehen somit in einem inneren Zusammenhang.

- Inhalt und Funktion der üblichen Hausaufgaben und Fördermaßnahmen müssen in diese Konzeption eingebunden sein.

- Gemeinsame und individuelle Freizeitgestaltung sind gleichrangige und wesentliche Aufgaben des Ganztagsschulkonzepts.

- Auf die Kostenträger kommen nicht unbeträchtliche Zusatzkosten für ein erweitertes Raum- und Personalangebot zu.

- Im Interesse der Betreuungsqualität muss die Kontinuität des Personals vormittags und nachmittags gesichert sein. 
- Ganztagsschule muss man lernen: die Lehrerbildung muss auf solche Anforderungen personell und konzeptionell vorbereitet sein. Deshalb darf, wer den Ganztagsschulbetrieb fordert, die Reform der Lehrerbildung in diesem Sinne nicht ausblenden.

Die Zusammenstellung macht deutlich, dass zu den genannten Voraussetzungen Versorgungsgesichtspunkte ebenso zählen wie Gestaltungsgesichtspunkte, häufig korrespondieren sie untereinander. Wenn wir überschlägig einmal durchrechnen, welche Aufwendungen erforderlich sind, um jede der ca. 40.000 Ganztagsschulen mit einer Kantine auszustatten, der hat eine ungefähre Vorstellung, was von den 4 Mrd., die die Bundesregierung für dieses Projekt bereitgestellt hat, dann noch übrig bleibt. Es sind noch keine Mehraufwendungen für die nachmittägliche Unterrichtssicherung und -gestaltung enthalten.

\subsection{Schulpädagogische Gestaltungsgrundsätze der Ganztags- schule}

Zwar erscheint in kontinuierlicher Folge Literatur zur Ganztagsschule, dennoch ist davon auszugehen, dass es eine Theorie der Ganztagsschule nicht gibt. Eine solche Theorie müsste sich historisch, systematisch, philosophisch-anthropologisch und gesellschaftsbezogen unter Einbeziehung empirischer Forschung, die es zu diesem Fragenkomplex nur rudimentär gibt, pädagogisch legitimieren. Das ist eine Aufgabe, die derzeit wenig Aussicht auf schnellen Erfolg hat und deshalb müssen Bemühungen um eine Substantiierung der Ganztagsschule derzeit mit dem Risiko leben, zufallsbedingt, interessengeleitet, parteilich, lückenhaft und perspektivlos zu agieren (vgl. Gauger Abschn. 9). Die Parteien Deutschlands verfügen über einen reichen Erfahrungsfundus in bildungspolitischem Handeln, das diese genannten Merkmale aufweist - die insgesamt doch unbefriedigend verlaufene und im Ergebnis höchst disparate Strukturdebatte der 70er Jahre und die Qualitätsdebatte der 90er Jahre mit 
z.T. desaströsen Befunden zu den Schulformen macht deutlich, auf welch dünnem Eis sich bildungspolitisches Handeln mitunter bewegt, wenn es nicht theoriegeleitet sich vollzieht, wenn es die Empirie in den Dienst der Politik zur Rechtfertigung regierungspolitischen Alltagshandelns zu stellen sich bemüht. Heinrich WOTTAWA (1981) hat vor mehr als 20 Jahren einschlägige Belege für den hier gekennzeichneten Zusammenhang aufgewiesen und ich bin sehr skeptisch, ob sich daran bis heute grundlegend etwas geändert hat.

Vor diesem Hintergrund sind die nachfolgend benannten Grundsätze zu bewerten.

- Ein nach meiner Einschätzung unabdingbarer Grundsatz ist die Gestaltung der Ganztagsschule als Angebotsschule. Zwei Modelle sind denkbar: Ganztagsschule als Angebotsschule neben anderen Schulformen (gebundene Ganztagsschule) oder Ganztagsschule als Angebotsschule, soweit es die Teilnahme an den nachmittäglichen Angeboten betrifft (offene Ganztagsschule). Je nachdem, welchem der beiden Modelle man nahe steht, sind die curricularen Folgen sehr weitreichend und sehr unterschiedlich.

In Variante 1 besteht Anwesenheitspflicht von 8.00 - 16.00 Uhr; Unterrichts- und Freizeitprogramm sind über den ganzen Tag verteilt, das gilt auch für obligatorische und fakultative Unterrichtsanteile; es gibt keine schriftlichen Hausaufgaben, das Mittagessen wird gemeinsam eingenommen.

In Variante 2 müssen die obligatorischen Unterrichtsanteile weiterhin am Vormittag unterrichtet werden, nachmittags folgen fakultative Unterrichtsanteile, das gemeinsame Mittagessen ist freiwillig.

- Die Ganztagsschule muss die zeitlich größere Präsenz der Schüler in der Schule als Chance sehen, die es im Sinne des eingangs genannten Schulzwe- 
ckes zu nutzen gilt und nicht als Belastung. Daraus folgt, dass sie ernst macht mit der - von der Schulkritik mal mehr mal weniger zutreffend eingeforderten - Pädagogisierung der Schule.

Aus diesem Grundsatz leite ich drei Folgerungen ab, die allesamt auf einen pädagogisch angemessenen Umgang mit Zeit zielen:

- Die Ganztagsschule ist von den äußeren zeitlichen Gegebenheiten in einer günstigen Ausgangslage, um auf die Veränderungen der Kindheit und der Jugend zu reagieren (Stichwort: Sozialpädagogisierung der Schule)

- Die Schule muss den Zuwachs an verfügbarer Zeit zur intensiveren individualen Betreuung jedes einzelnen Schülers einsetzen. Hier liegt eine zweifach realistische Chance für einen zumindest partiellen Ausgleich

$\Leftrightarrow$ der Nachteile, die von großen Lernverbänden auf die Unterrichtsqualität zurückwirken können;

$\rightarrow$ der familiaren Funktionsverluste. Hier liegen allerdings auch Risiken, der Demotivation vieler Schüler entgegenzuwirken;

(Stichwort: Individuation der Betreuung)

- Der Zugewinn an Zeit muss insbesondere in der Ausgestaltung des Schullebens erfahrbar werden. Dazu gehören u.a.:

Außerschulische Projekte und Workshops

> Multikulturelle Begegnungen

Mitarbeit an Schul-/Schülerzeitungen

\section{aber auch:}


Auffangen und Verarbeiten von Störungen

Entwickeln von Gesprächskultur und Dialogfähigkeit

Aufarbeitung von Fehlern im Bereich von Lernen und Leisten, von sozialem Verhalten, von intergenerativer Kommunikation etc.

\section{$4.5 \quad$ Fazit}

Das hier aufgezeigte Profil setzt eindeutig auf die Umsetzung des eingangs genannten Schulzweckes insgesamt. Die Schule ist ohne Einschränkung Unterrichtsort, aber sie eröffnet auch Möglichkeiten der pädagogisch geleiteten Erschließung der Lebenswelt und der Gestaltung des Schullebens. Diese Zieldimension ist gemeinsames Fundament aller Schulen, der Halbtagsschule wie der Ganztagsschule. Ersterer wird gelegentlich unterstellt, dass dort beide Teildimensionen - Bildung und Erziehung gelegentlich nur eingeschränkt zur Geltung kommen. Die herangezogenen Gründe dafür sind vielfältig:

- Straffe stoffliche Gliederung

- Unterrichtsausfall

- Unterfinanzierung

- $\quad$ ungünstige Lehrer-Schüler-Relation - Unterversorgung mit Personal

- $\quad$ einseitige Akzentuierung von Bildung oder Erziehung

- edukative „Enthaltsamkeit“ von Elternhaus oder Schule

- etc.

Es ist meines Erachtens eine empirisch offene Frage, ob die notierten Mängel tatsächlich zwischen der Ganztags- und der Halbtagsschule zu verteilen sind. So trifft Unterfinanzierung die Schulen unabhängig von ihrer Struktur, also unabhängig vom Ganztags- oder Halbtagsbetrieb; Einseitigkeiten sind sehr stark personenabhängig 
und deshalb in beiden Systemen beobachtbar. Deshalb ist die Ausweitung des Ganztagsbetriebes wissenschaftlich zu begleiten, um Wildwuchs zu vermeiden und der Kritik zu begegnen, die durch die Datenlage nicht belegbar ist. Das trifft im Falle der Ganztagsschule auf große Schwierigkeiten, ist sie doch durch das Bundesministerium für Bildung und Forschung publizistisch instrumentalisiert, wie andere „Aktionismen“ dieses Hauses in den vergangenen Jahren auch, z.B. die Kampagne „Ein Laptop für jeden Schüler“, die Juniorprofessur, das Verbot von Studiengebühren etc. etc. Das Investitionsprogramm „Zukunft Bildung und Betreuung“ (IZBB) der Bundesregierung ist solange ein „Lockvogelangebot“, wie es die horrenden Folgekosten der Einrichtung von Ganztagsschulen, insbesondere die Personalkosten bei den ohnehin finanzpolitisch „klammen“ Ländern und Kommunen belässt, ohne Kompensationsangebote zu unterbreiten - immerhin reden wir über ca. 30 \% höhere Kosten gegenüber den ohnehin unterfinanzierten Halbtagsschulen.

Die verantwortliche Politik ist, wie wir meinen, gut beraten, wenn sie Fehler, die von allen Parteien bei der Reform der Schule und des Bildungswesens insgesamt gemacht wurden, nicht wiederholt. Dabei kann man punktuell durchaus vom jeweils anderen lernen. Ohne nun die „Sünden“ der jeweils anderen Seite aufzurechnen, kann man die in den 70er und 80er Jahren mit geradezu missionarischem Eifer in den alten Bundesländern geführte, im Ergebnis aber nicht konstruktive Strukturdebatte als eines jener Konfliktfelder bezeichnen. Um Missverständnissen im Vorfeld zu begegnen: Der Diskurs über die Struktur des Schulwesens ist notwendig und wichtig, weil er den Sachverstand von ideologischen Überformungen zu entlasten geeignet ist. Die Strukturdebatte darf aber die nicht minder wichtige substantielle Innovation der Schule zurückdrängen. In unseren Schulen herrscht auch heute noch - zwar nicht überall, aber weit verbreitet - methodisch-didaktischer Monismus. Es gibt eine Monokultur der Unterrichtsgestaltung, die von der Vielfalt der didaktischen und methodischen Gestaltungsmöglichkeiten kaum Gebrauch macht. Diese Lehre aus TIMSS und PISA haben wir bisher nicht gezogen. 
Einer der Fehler, den wir im Umgang mit der Ganztagsschule nicht wiederholen sollten, ist die Art der öffentlichen Auseinandersetzung, wie sie beispielsweise bei der Debatte um die Gesamtschule ablesbar ist. Die Art der Auseinandersetzung klärte weniger als sie belastet. Das Hantieren mit durchaus griffigen Etiketten, die einer flächendeckenden Akzeptanz in der Bevölkerung sicher sein können, mit denen man im übrigen auch Wählerstimmen und damit auch Wahlen gewinnen kann, ist wohl geeignet, das politische Terrain zu ebnen - das Beispiel der rheinland-pfälzischen Ganztagsschulkampagne vor der Landtagswahl des Jahres 2001 ist noch in lebhafter Erinnerung. Heute wird dieser Widerspruch zwischen Verpackung und Inhalt, zwischen dem griffigen sprachlichen Etikett und dürftiger schulpädagogischer Operationalisierung erkennbar und die Politik rudert zurück, zwar nur zentimeterweise, aber für den aufmerksamen Beobachter doch wahrnehmbar.

Der Debatte um die Ganztagsschule ist zu wünschen, dass sie weniger auf Populismus und mehr auf Sachauseinandersetzung setzt - im Bund trotz seiner begrenzten Zuständigkeit, in den Ländern allemal, weil dort die Entscheidungen fallen. Die Reformansätze der letzten Jahre haben den Blick auf die zentralen Aufgaben der Schule zuweilen verstellt. Immer mehr und immer neue Funktionen sind ihr als Folge des Funktionsverfalls anderer Erziehungsinstanzen aufgebürdet worden, ohne zuvor eine Klärung herbeizuführen, ob die neuen Aufgaben mit dem Zweck der Bildungseinrichtung Schule vereinbar sind. Insbesondere im Kontext mit Ganztagsschulen gibt es auch durchaus ernst zu nehmende kritische Anmerkungen, die aber untergehen oder nicht kommuniziert werden, weil sie politisch nicht opportun sind: So hat Helmut FEND (1982, 262 ff, vgl. auch Abschn. 15.5 dieses Bandes) bereits darauf verwiesen, dass in Ganztagsschulen nicht - wie angenommen - die leistungsschwächeren Schüler durch Ausgleich fehlender häuslicher Unterstützungssysteme besser werden, sondern vielmehr die leistungsstärkeren durch den scheinbaren Wegfall dieser häuslichen Unterstützungssysteme im Zuge der Rückverlagerung schulbezogener Tätigkeiten in die Schule im Leistungsniveau absinken. Das heißt: Insgesamt verringert sich die Lernzeit besonders für Schüler, die im halbtägig geführten Schulsystem 
zu Hause viel für die Schule gelernt haben. Für Schülergruppen, die auch im halbtägig geführten Schulsystem eher wenig außerhalb des Unterrichts gelernt haben, ist somit auch keine Veränderung der Lernzeiten und damit auch nicht der Schulleistungen erwartbar.

Damit holt uns die empirische Bildungsforschung auf den Boden der Tatsachen zurück. Um diese realistische Bodenhaftung bemühen wir uns im Schlusskapitel dieses Bandes (HANSEL, Abschn. 15). Nicht der euphorisch getrübte Blick auf ein für Deutschland gar nicht so neues System, sondern die ergebnisorientierte Analyse des vorhandenen Wissens über dieses System ist angesagt - vorurteilsfrei und entideologisiert, damit die Debatte über die Ganztagsschule nicht so endet wie die Strukturdebatte, auf die oben verwiesen wurde: wortgewaltig und qualitätsmindernd.

\subsection{Literatur}

FEND, Helmut: Gesamtschule im Vergleich. Bilanz der Ergebnisse des Gesamtschulversuchs, Weinheim/Basel 1982

GIESECKE, Herrmann: Brauchen wir mehr Ganztagsschulen? Funkmanuskripte Bd. 8, Göttingen 2004

Holtappels, Heinz Günter: Ganztagsschule und Schulöffnung, Weinheim/München 1994

Holtappels, Heinz Günter/SCHNETZER, Thomas: Analyse beispielhafter Schulkonzepte von Schulen in Ganztagsform, Institut für Schulentwicklungsforschung, Dortmund 2003

LUDWIG, Harald: Entstehung und Entwicklung der modernen Ganztagsschule in Deutschland, Bd. 1 und 2, Köln/Wien/Weimar 1993

WOTTAWA, Heinrich: Die Kunst der manipulativen Berichtlegung in der Evaluationsforschung, in: Zeitschrift für Entwicklungspsychologie und Pädagogische Psychologie, Bd. XIII Heft 1, 1981/S. 45-60 